and escape of neutrinos by the photoneutrino, pair annihilation, and plasma neutrino mechanisms.

The neutrino energy loss results in a phase of rapid collapse $\left(\sim 5 \times 10^{3} \mathrm{yr}\right)$ when the neutrino and optical luminosities as well as the effective temperatures are high $\left(L \sim 10^{3} L_{\odot}, L \nu \sim 10^{2} L, \log T_{\text {eff }} \sim 5\right)$.

A comparison with recent work by Harmon and Seaton (Astrophys. J. 140, 824, 1964) suggests the identification of these tracks with their proposed evolution for the nuclei of planetary nebula. This might be used as an argument for the reality of the theory of a universal weak interaction (Feynmann and Gell-Mann, Phys. Rev. 109, 193, 1958; Sudarshan and Marshak, Phys. Rev. 109, 1960, 1958) responsible for the above neutrino reactions.

The Solar Rotation Rate, Sunspot Development, and the General Circulation of the Solar Atmosphere. Frederick W. Ward, Jr., Air Force Cambridge Research Laboratories.-The analysis of daily sunspot group proper motions for the 50-yr period 1905-1954 reveals three fundamental features of the horizontal wind field in the sunspot layer of the solar atmosphere. The large-scale or general circulation is fundamentally of a non-axisymmetric type with significant longitudinal and temporal changes. The time and/or space eddy wind field transports angular momentum equatorward in both hemispheres at a rate equal to $2 \%$ per day of the excess angular momentum in equatorial regions on the average. This process therefore appears to be the fundamental mechanism whereby the "equatorial acceleration" is maintained.

The average east-west motion, or rotation rate, is shown to be an inverse function of spot size. On the basis of some theoretical arguments, verified by data from the terrestrial atmosphere, it seems reasonable to assume the value derived from the smallest identifiable features to be the correct estimate of mass motions. On this basis, the solar rotation rate should be revised upward by $1-2 \%$ at all latitudes between $0-35^{\circ}$.

The east-west proper motion of sunspot groups decreases as the group develops in area. A similar situation exists for cyclonic storms in the terrestrial atmosphere and is a fundamental part of the energy exchange picture of the general circulation. It appears reasonable to assume a similar situation exists in the solar atmosphere.

Absorption Spectrum of $\mathrm{Fe} I$ in the Vacuum Ultraviolet. DoRothy W. WeEks, Harvard College Observatory.-Absorption spectrograms of $\mathrm{Fe} \mathrm{I}$ in the region 2428 to $1650 \AA$ in the temperature range $1500^{\circ}$ to $2500^{\circ} \mathrm{C}$ were obtained by using the carbon- tube furnace. Likewise, absorption spectrograms of $\mathrm{Fe} \mathrm{I}$ in the region 1998 to $1460 \AA$ were obtained by using the shock tube. Forty-three known lines of $\mathrm{Fe} \mathrm{I}$ were used as standards between 2000 to $1855 \AA$, and for the shorter wavelengths on the furnace plate 16 predicted lines of Fe I and six Fe II lines were separately used as wavelength standards. From an analysis of the 300 spectral lines in the region $1998 \AA$ to 1750 common to both spectrograms it appears that 21 new odd energy levels have been found, representing 269 lines, 88 lines previously observed in the laboratory and 181 lines observed in the solar spectrum 54 of which had not been identified.

Interstellar Lines and the Dissipation of Interstellar Magnetic Fields by Petschek's Mechanism. Donat G. Wentzel, The University of Michigan.-An efficient method of dissipating magnetic fields has been proposed by Petschek (The Physics of Solar Flares, NASA SP-50, p. 425, 1964). If such dissipation occurs within an interstellar cloud which radiates efficiently, it generates a thin layer of gas with a density of roughly 100 atoms $/ \mathrm{cm}^{3}$ and a streaming speed of $\pm 3 \mathrm{~km} / \mathrm{sec}$. This inhomogeneity appears in 21-cm emission as a symmetrical pattern in the sky or as a Doppler profile with several, probably three, resolvable components. It is more visible in $21-\mathrm{cm}$ absorption because the dense layers are cooled efficiently. The inhomogeneity may actually dominate the absorption lines of $\mathrm{Ca}^{+}$and $\mathrm{Na}^{\circ}$, the abundances of which are proportional to the density. Observed ratios of line strengths (Howard, Wentzel, and McGee, Astrophys. J. 138, 988, 1963) can be made consistent with a uniform abundance of free $\mathrm{Ca}$ and $\mathrm{Na}$ in low-velocity clouds; the underabundances relative to the sun become a factor 300 for $\mathrm{Ca}, 6$ for $\mathrm{Na}$.

In analogy to the geomagnetic field, Petschek's mechanism may disconnect interstellar $\mathrm{H}$ I clouds from the general galactic field. If so, clouds move independently of each other and of the galactic field; consequently spiral arms must be gravitational in origin and the galactic field, constrained only by negligible intercloud mass, must be nearly force-free. An elongation of the clouds along the confining galactic field can explain the alignment of interstellar polarization. The evolution of clouds may consist of their formation near a supernova, dissipation of internal magnetic fields and a compensating increase in density during some $10^{8} \mathrm{yr}$, and finally star formation or simply disintegration.

Mapping the Brightness-Temperature Distribution of Jupiter at $10 \mu$. RoBERT L. WILDEY, Mount Wilson and Palomar Observatories; Division 
of Geological Sciences of the California Institute of Technology; and U. S. Geological Survey Branch of Astrogeology.-The 8-14 $\mu$ infrared emission of Jupiter has been observed on six nights in December of 1963 using the 200-in. Hale telescope. The new observations possess twice the resolution of those obtained in 1962. The brightness temperature at the center of the disk appears to be closely constant at $129^{\circ} \mathrm{K}$. With some slight ambiguity, the light bands are about 0.5 cooler in appearance than the dark bands. There is some suggestion of morning-evening asymmetry in one of the bands. The Great Red Spot is found to be from 1.5 to $2: 0$ cooler than the surrounding disk at the newer resolution.

The observations were collected with a photometer employing a mercury-doped germanium photoconductor utilized as a $180 \mathrm{cps}$ transducer through the use of a simple articulated double mirror spatial filter which eliminates a large portion of sky-telescope background from the signal.

The present limitations to these maps arise from the nonstationary nature of part of the noise. In the future, noise-suppressed maps may be possible without superposition of several maps, which may then remove some of the ambiguity of the present interpretation.

These observations will be presented in greater detail in the current issue of the Journal of Geophysical Research.

\section{Measurement of the Flux of Five Sources at} $4080 \mathrm{Mc} / \mathrm{sec}$. R. W. Wilson and A. A. Penzias, Bell Telephone Laboratories, Inc.-The receiving system employed in the absolute flux measurement of Cas A at $4080 \mathrm{Mc} / \mathrm{sec}$ in the fall of 1964 (Penzias, A. A., and Wilson, R. W., submitted to Astrophysical J.) has been used to measure the flux of Cygnus A, Taurus A, Orion A, and Virgo A. The accuracy of the present measurement is $3 \%$ probable error.

The flux of Cas A has been remeasured as a check of system stability. The night-to-night variation in the measured flux density of each source is found to be less than $\pm 2 \%$.

Correcting the observed values for source size and atmospheric absorption the following flux densities were found (units of $10^{-26} \mathrm{~W} \mathrm{~m} \mathrm{~m}^{-2} \mathrm{cps}^{-1}$ ): Cas $\mathrm{A}$, 1080; Cyg A, 476; Tau A, 711; Ori A, 445, and Vir A, 87.1.

Automatic Measurement of Radial Velocities. Kenneth M. Yoss and Thomas E. Lutz, University of Illinois. - The plate positions for central depths of stellar lines and peaks of comparison lines are recorded with a digitized microphotometer. Two scans are necessary, with the stellar and comparison lines alternately masked at the microphotometer slit. The digitized data are processed in an electronic computer, following standard procedure. The plates measured were obtained at Mount Wilson Observatory, with the 60-in. reflector and $\mathrm{x}$-spectrograph $(40 \AA / \mathrm{mm})$. The spectral and wavelength ranges considered are G8 to K3 and 4000 to $4400 \AA$, respectively. Approximately 45 iron lines are used for establishing the dispersion curves.

Line blending necessitates the establishment of effective wavelengths for the stellar lines, which generally depend on spectral type, dispersion, and method of measurement. Seven spectrograms of five I.A.U. standard radial velocity stars were measured for this purpose. The plate positions for the stellar lines were reduced to zero radial velocity, yielding effective wavelengths. Approximately 50 lines are found to be suitable, giving an internal mean error in radial velocity of approximately $\pm 10.0 \mathrm{~km} / \mathrm{sec}$ for a single line and $\pm 1.4 \mathrm{~km} / \mathrm{sec}$ for a single plate. By comparison, conventional measurements for this dispersion give approximately $\pm 5.2 \mathrm{~km} / \mathrm{sec}$ for a single line. However, usually fewer lines are measured per plate, giving a plate mean error comparable to the value found here.

Of the 18 lines listed by Wright (Wright, K. O., Publ. Dom. Astrophys. Obs. Victoria 9, 167, 1951) in the wavelength and spectral ranges considered, for which single effective wavelengths are valid, 14 are useful for the present method of measurement. The mean difference, Wright minus present values, is $-0.04 \pm 0.02 \AA$, suggesting no major systematic difference between the preliminary values established here for $40 \AA / \mathrm{mm}$ and the values of Wright for 30 $\AA / \mathrm{mm}$. However, several individual lines differ by as much as $0.15 \AA$, illustrating the necessity of a new set of effective wavelengths.

Glass-Filter Temperature Effects in Astronomical Photometry. ANDREW T. Young, Harvard College Observatory.-The effects of temperature on colored glass filters have long been known [K. S. Gibson, Phys. Rev. 7, 194, 1916; S. Meyer, Glastech. Ber. 14, 305, 1936; A. J. Holland and W. E. S. Turner, J. Soc. Glass Tech. 25, 164, 1941 ; A. K. Angstrom and A. J. Drummond, Quart. J. Roy. Meteorolog. Soc. 85, 429, 1959; J. R. Hickey, "A Study of Radiometric Filters" (M.S. thesis, University of Rhode Island, 1962)]. For example, the cutoff wavelength of Schott GG 13 and GG 11 and Corning 3384 shift by $0.8,1.2$, and $1.1 . \AA /{ }^{\circ} \mathrm{C}$, respectively. The temperature coefficient of cutoff lies between 1 and $2 \AA /{ }^{\circ} \mathrm{C}$ for glasses cutting in the visible spectrum, and is greater at longer wave- 